\title{
On the interaction of the evaporation and the thermocapillary convection in capillary tubes
}

\author{
Khellil Sefiane $^{1, a}$, Rachid Bennacer $^{2}$ and Mohammed El Ganaoui ${ }^{3}$ \\ 1 School of Eng. Electronics, University of Edinburgh, Kings Building, Edinburgh, EH9 3JL, UK \\ 2 LEEVAM-LEEE, Université de Cergy Pontoise, 95031 Neuville sur Oise, France \\ 3 SPCTS, UMR CNRS 6638, Faculté Sciences et Tech., Université de Limoges, 87000 Limoges, France
}

Received 10 October 2003, Accepted 3 February 2004

\begin{abstract}
With the increasing demand for improved electronics cooling, thermal management and cooling techniques have evolved in design and efficiency over the last three decades. The paper focuses on heat pipes as practical as possible inexpensive alternatives to cool electronic components. An analysis of the necessary conditions allowing the appearance of convective motion and its interaction with energy exchange at the liquid/vapour interface is performed. The numerical simulation allows the access to the evaporation profile and its dependence with the operating conditions. The role of the inversion of the thermal distribution on the development and the orientation of the Marangoni cells and the local convection near the contact line is presented.
\end{abstract}

Key words: Capillary tube / evaporation / numerical simulations

Résumé - Sur l'interaction de l'évaporation et la convection thermocapillaire dans les tubes capillaires. Une revue des techniques de refroidissement des composants électroniques à haute puissance est présentée en précisant le rôle que peuvent jouer les tubes capillaires pour les alternatives futures. L'étude introduit une investigation de l'évaporation dans des tubes capillaires de liquides volatiles. Une approche numérique a permis d'étudier le rôle de l'évaporation dans l'initiation du mouvement de convection dans la phase liquide. Une convection thermocapillaire peut se développer selon le profil d'évaporation imposé le long de l'interface liquide/vapeur. La compréhension de ce mécanisme est très utile pour l'amélioration des performances des tubes capillaires à une échelle microscopique.

Mots clés : Tube capillaire / évaporation / simulations numériques

\section{Introduction}

The development of the microprocessor in the 1970's by the Intel Corporation marked a beginning in the microelectronics industry. However, when high concentration of thousands or even millions of components are packed in a microprocessor (less than $10 \mathrm{~mm} \times 10 \mathrm{~mm} \times 1 \mathrm{~mm}$ ), the generated heat increases to such high levels that its removal has become a formidable task and a major concern for the safety and reliability of electronic devices Azar [1]. A US Air Force survey confirmed that more than $50 \%$ of all electronics failures are related to overheat. The trend towards miniaturization and high-density packing in microelectronics components has placed a large demand on associated cooling systems Berestecky [2].

\footnotetext{
a Corresponding author: ksefiane@ed.ac.uk
}

Figure 1 shows how module heat fluxes have increased during the last decade. This overall trend is in agreement with Moore's prediction that semiconductor density and performance doubles roughly in 18 months. In 1990, bipolar chip heat fluxes of up to $15 \mathrm{~W} / \mathrm{cm}^{2}$ were reported. This can lead to very high chip surface temperatures if conventional air-cooling or indirect liquid-cooling systems are used. For example, a typical $3 \mathrm{~mm} \times 3 \mathrm{~mm}$ chip with a total thermal resistance of $80^{\circ} \mathrm{C} / \mathrm{W}$ can lead to a temperature difference of $72^{\circ} \mathrm{C}$ between the chip surface and ambient, if the dissipated heat flux reaches $10 \mathrm{~W} / \mathrm{cm}^{2}$. This would result in a chip surface temperature of $100^{\circ} \mathrm{C}$ where the ambient temperature is $28^{\circ} \mathrm{C}$. The failure rate of electronic devices increases rapidly at such temperatures. With the development of circuit technology, bipolar chip surface heat flux up to $100 \mathrm{~W} / \mathrm{cm}^{2}$ and CMOS chip heat flux up to $50 \mathrm{~W} / \mathrm{cm}^{2}$ have been projected for 


\section{Heat Flux Explosion}

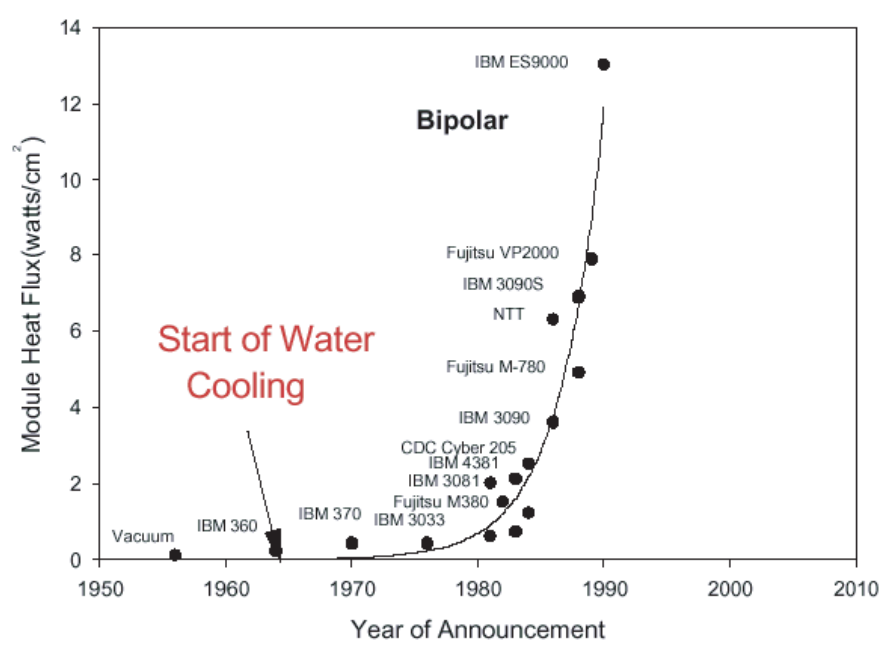

Fig. 1. Heat flux explosion in microelectronics thermal management.

the year 2010. Clearly power dissipation has become the major bottleneck for development of these smaller, faster and cheaper chips Chu [3].

To meet the increasing demand for high power dissipation in microelectronics, concepts including micro-fans on chips, rotating-stirrer cooling, curved-channel cooling, capillary-pumped loops, micro heat pipes and flow boiling in micro channels, have been proposed and investigated at their early stages. However, the issues of cost, reliability and maintenance have to be addressed before their potential could be further developed Garner [4].

Various cooling techniques have been introduced, depending on heat transfer mechanisms and coolants utilized. It is noted that principally, the highest heat transfer rate can be achieved with phase change processes, although these processes are involved with complicated systems and thus reliability issues.

Natural convection with single-phase flows will lead to very low heat transfer performance. However, cooling techniques with natural convection are still attractive in applications with low dissipating heat fluxes, due to simplicity, low cost, and reliability of such techniques. Single-phase forced convection offers higher heat transfer rates with extra costs for fans or pumps. The cooling performance of these systems is largely influenced by the reliability of the fans or pumps. Basically such systems could cope with moderate power dissipation. Thus it is suggested that for high heat flux power dissipation, twophase flow and heat transfer mechanism should be considered, although complex circulating loops are required. Nevertheless, for low power dissipation, simple cooling techniques should be used.

Heat pipes can be practical and inexpensive alternatives to cool electronic components at moderate heat fluxes. A heat pipe is a device without moving parts and power input, that can transfer large amounts of heat from the evaporating end to the condensing one using a small

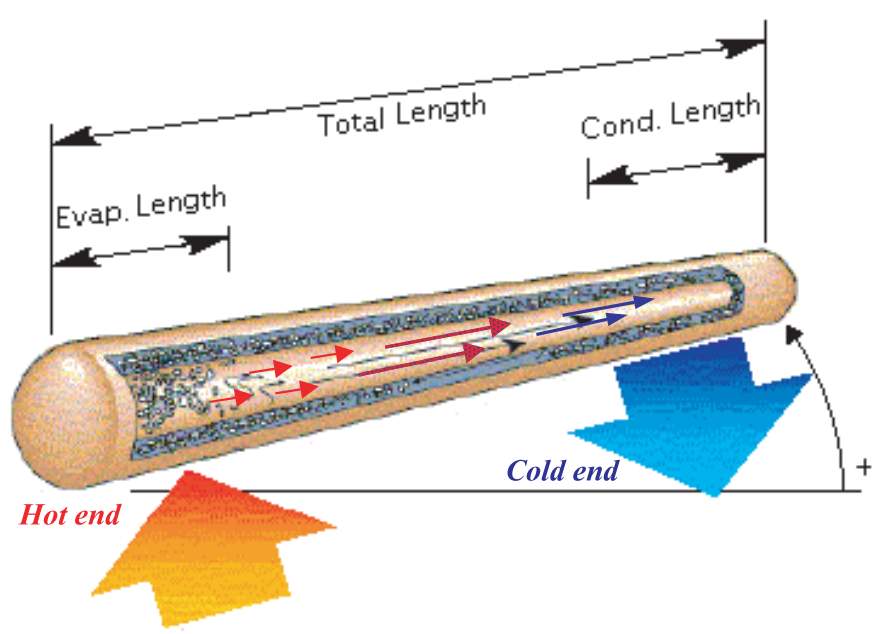

Fig. 2. Principle of the heat pipe.

temperature difference $\left(1\right.$ to $\left.5{ }^{\circ} \mathrm{C}\right)$ between the two ends. Heat pipes use the advantages of phase change and capillary force transportation to achieve high heat transfer rates. The structure and operating principles of a heat pipe are illustrated in Figure 2. Heat pipes have an effective conductivities several hundred times that of copper or silver. For instance, a simple heat pipe with water as the working fluid has an effective thermal conductivity of the order of $10^{5} \mathrm{~W} / \mathrm{m}^{\circ} \mathrm{C}$ compared with about $400 \mathrm{~W} / \mathrm{m}^{\circ} \mathrm{C}$ for copper. The basic shape of heat pipes is a cylindrical vessel. However, heat pipes with various shapes such as $90^{\circ}$ bends, S-turns, spirals, and flat plates, have been developed.

The performance of a heat pipe is influenced by many factors. The operating temperature determines the type of fluid and the operating pressure inside the heat pipe. Water is a suitable fluid to use in the moderate temperature range encountered in electronic equipment. The inner pressure can control the operating temperature at which water undergoes a phase-change process. In fact, candidate fluids should have a high surface tension to enhance the capillary effect and be compatible with the wick and tube materials, as well as being readily available, chemically stable, non-toxic, and inexpensive.

The operating heat load must be under the value at which film boiling or boiling crisis occurs at the hot end. At the transition point, the return of the condensate can be hindered and operation will cease. The structure of the wick, usually porous ceramic material or woven stainless wire mesh, provides the means for the return of the liquid to the evaporator, and therefore has a strong effect on the heat pipe performance. The optimum pore size of the wick will be determined by fluid properties and pipe orientation. An improper wick will yield an inadequate liquid supply and eventual failure of the heat pipe. In the gravity field, the orientation in which the capillary and gravity forces act in the same direction will yield the best performance. The worst heat transfer rate will occur when the two forces act in opposite directions. 
Numerous investigations on heat pipes have been reported in the literature. The publications related to electronics cooling have covered a range of studies. Typically, Garner [4] analysed heat pipes in electronic cooling applications. He summarized that heat pipes provide much higher capacity, compared to metallic heat sinks with fans. He also highlighted five primary problems for heat pipes applied in electronics cooling, i.e. viscosity, sonic velocity, capillary pumping, entrainment or flooding, and vigorous boiling. These limitations are caused by a number of operating conditions. For example, a low operating temperature may lead to high viscous forces hindering the vapour flow in the heat pipe. The effective thermal resistance of a heat pipe depends on the geometry, evaporator length, condenser length, wick structure, and working fluid. For a typical copper/water heat pipe, heat transferred is a function of temperature difference between the evaporator and condenser. It is seen that the heat-pipe sink can dissipate heat loads of $100 \mathrm{~W}$ at heat resistance of 0.2 to $0.4{ }^{\circ} \mathrm{C} / \mathrm{W}$. The equivalent heat flux at the evaporator is about $4 \mathrm{~W} / \mathrm{cm}^{2}$.

Graebner [5] tested a heat pipe, $6.35 \mathrm{~mm}$ in diameter and $300 \mathrm{~mm}$ in length. He showed that at a heat load of $72 \mathrm{~W}$, the evaporating end becames very hot and unstable. He found that the temperature difference between the hot and cold end rose with increasing power and the maximum power transferred was determined by the orientation, working fluid and wick construction.

It is clear that heat pipes offer much higher heat transfer rate, compared to may other cooling techniques. They can practically operate in an isothermal fashion and are relatively compact. However, heat pipes become unstable and lose their low thermal resistance at high power levels or after long periods of operation. For most cases, heat pipes operate at fluxes below $20 \mathrm{~W} / \mathrm{cm}^{2}$. Another major obstacle for their application in electronics cooling is the sudden drop of effective heat transfer rate due to overload pulses or capillary structure degrading. This can lead to a serious damage of electronic devices.

It is established experimentally that thermocapillary convection related to interfacial conditions takes place when volatile liquids evaporate from capillary tubes into air Sefiane [6]. Indeed interfacial conditions are dictated by the various heat and mass fluxes operating during the process. Such typical coupled problem, drawn in Figure 3, involves heat conduction in the liquid phase towards the interface and inside the solid wall. At the liquid/vapour interface, the heat flux corresponding to the phase change must satisfy both the energy and the species conservation. Conduction in the vapour phase could be neglected because of the weak thermal conductivity. The generated vapour is transported from the interface to the bulk gas by diffusion. Because the meniscus wedge is near the solid wall and because of the cylindrical configuration, larger heat flux is transferred to the closer fluid than to the centre resulting in higher evaporation rate near the contact line. This non-uniform evaporation rate induces non-uniform temperature distribution on the interface. Since surface tension for most liquids is a decreasing

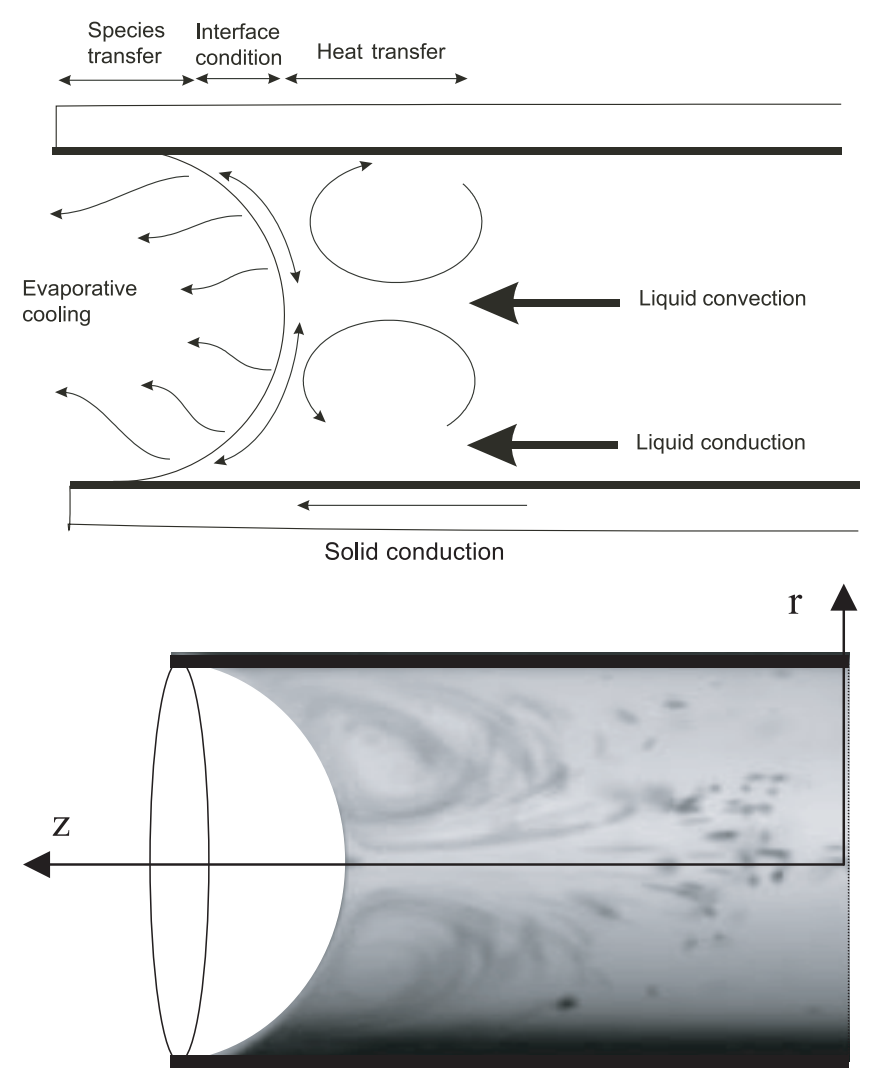

Fig. 3. Experimental result showing thermocapillary convection rolls.

function of the temperature, a surface tension gradient along the interface is hence generated. This thermocapillary stress will drive a convective motion in the liquid phase. Fluid moves from low surface tension (high temperature) to high surface tension (low temperature) as observed experimentally by Molenkamp [7]. Thermocapillary convective cell will bring hot liquid from the bulk altering the interfacial temperature profile, which will influence the evaporating rate. The present study presents a non-conventional Marangoni flows because arising from evaporation; thermal energy is removed from the liquid resulting in local changes in the temperature and thus in surface tension. This mechanism has been investigated and discussed under other conditions in many recent references (Ruiz et al. [8]). Whilst it is experimentally difficult to access to the local evaporation rate, the numerical simulation can be an effective tool to describe the controlling parameters. The present results focus on the conditions allowing the apparition of convective motion and its dependancy on energy exchange at the $\mathrm{L} / \mathrm{V}$ interface.

\section{Modelisation and simulations}

\subsection{Computational method}

In order to avoid the roughness of the tube and the contact angle effect, a planar interface is considered to focus on the interaction between the thermal conditions 


\begin{tabular}{llll}
\hline$r=0$ (sym. axis) & $r=1$ (solid wall) & $z=A(\mathrm{~L} / \mathrm{V}$ interface) & $z=0$ \\
\hline$\frac{\partial T}{\partial r}(0, z)=0$ & $T(1, z)=1$ & $\frac{\partial T}{\partial z}(r, A)=q(r)$ & $\frac{\partial T}{\partial z}(r, 0)=0$ \\
$\frac{\partial u_{z}}{\partial r}(0, z)=0$ & $u_{z}(1, z)=0$ & $u_{z}(r, A)=P e$ & $u_{z}(r, 0)=P e$ \\
$u_{r}(0, z)=0$ & $u_{r}(1, z)=0$ & $\frac{\partial u_{r}}{\partial z}(r, A)=-M a \frac{\partial T}{\partial r}(r, A)$ & $\frac{\partial u_{r}}{\partial z}(r, 0)=0$ \\
\hline
\end{tabular}

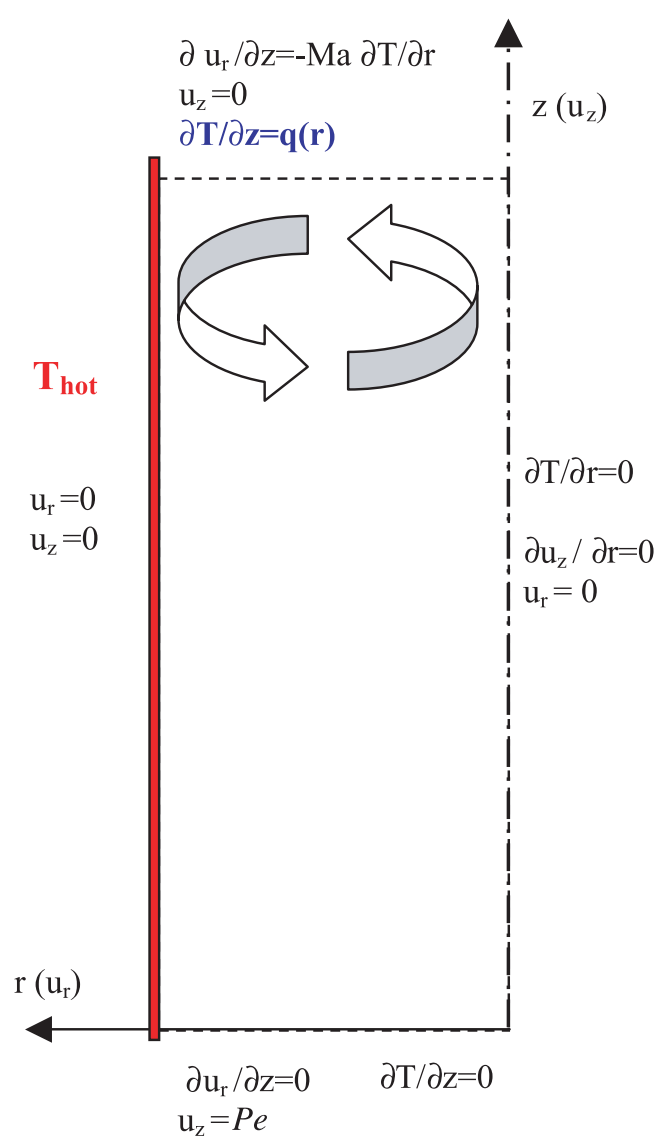

Fig. 4. Schematical of the considered domain for computationnal.

on the interface and the convection appearing in the liquid phase. The cylindrical domain of radius $R$ and length $L$ (Fig. 4) is considered. Only the axially symmetrical steady state are cheked. The flow is assumed incompressible and laminar for a Newtonian fluid. Physical properties (density $\rho$, shear viscosity $\mu$, thermal diffusivity $\alpha$ ) are assumed constant and the surface tension $\sigma$ decreases linearly with the temperature $\left(\gamma=\frac{\partial \sigma}{\partial T}<0\right)$.

The solid wall is maintained at temperature $T_{0}$. On the free surface a permanent heat flux is imposed, $Q(r)=$ $Q_{0} q(r)$, where $q(r)$ is a dimensionless function of the axial coordinate $r$ and $Q_{0}$ the constant flux case considered as a reference heat flux used to define the dimensionless temperature. Constant, polynomial and Gaussian $q(r)$ distributions are used to model different evaporation rate at the free surface.
We use $R, \alpha / R^{2}, \rho \alpha^{2} / R^{2}$ and $Q_{0} R / \lambda$ respectively as the scale factors for the length, time, pressure and temperature. Under no gravity conditions, the control parameters are the Prandtl number, $\operatorname{Pr}=\nu / \alpha$ and the Marangoni number $M a=-(\partial \sigma / \partial T) \Delta T_{R} / \mu \alpha$.

Boussinesq equations in a non-dimensional form are:

$$
\begin{aligned}
\nabla \cdot u & =0 \\
\frac{\mathrm{d} u_{r}}{\mathrm{~d} t} & =-\frac{\partial p}{\partial r}+\operatorname{Pr}\left[\Delta u_{r}-\frac{u_{r}}{r^{2}}\right] \\
\frac{\mathrm{d} u_{z}}{\mathrm{~d} t} & =-\frac{\partial p}{\partial z}+\operatorname{Pr} \Delta u_{z} \\
\frac{\mathrm{d} T}{\mathrm{~d} t} & =\Delta T
\end{aligned}
$$

where $\frac{\mathrm{d}}{\mathrm{d} t}=\frac{\partial}{\partial t}+u \nabla$ and $\Delta=\frac{\partial}{r \partial r}\left(r \frac{\partial}{\partial r}\right)+\frac{\partial}{\partial z^{2}}$.

The dimensionless boundary conditions for the investigated problem are summarized above.

The problem is solved in the dimensionless domain $[0,1] \times[0, A](A=L / R$ is the aspect ratio $)$. In this work, $\mathrm{Pr}=10$ and high aspect ratio are tested to simulate a long tube, the ratio $A=5$ is found to be sufficient, for a range of $M a$ and slope and shape of the axial cooling.

A control volume approach, Patankar [9], is used to discretize the governing Equations (1)-(4) associated to boundary conditions. The resulting algebraic system is solved using an ADI method coupled to a block correction. The pressure-velocity interlinking is solved using the SIMPLE algorithm. The advection-diffusion terms are approximated using a second-order centered scheme. A non-uniform grid of at least $161 \times 61$ nodes is used for most computations presented in this paper, for details see Bennacer et al. [10].

\subsection{Numerical results}

Strong thermocapillary convection was observed to be resulting from evaporation (Fig. 3), the considered sizes $(R<100 \mu \mathrm{m})$ in the present study allow us to neglect the Rayleigh convection. The orientation of the convective cells is found to be dependent on the heat flux distribution along the meniscus. In fact the real driving force for convection is the tangential capillary stress at the interface generated by the interfacial temperature gradient. This gradient is itself influenced by the convection in the 


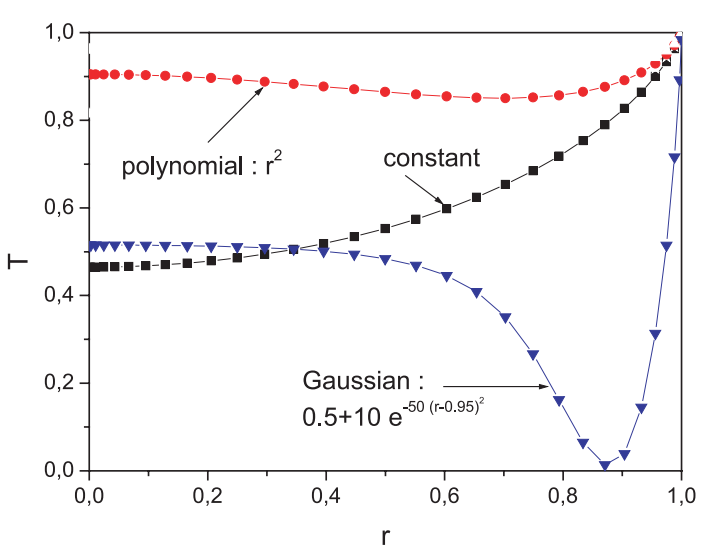

Fig. 5. Thermal response at the Liquid Vapour interface to the applied evaporating rate.

bulk liquid. Indeed the thermocapillary convection tends to bring hotter liquid from the bulk toward the interface increasing the temperature at the center and consequently the strength of the convection. The local evaporation rate is dictated by the local vapor pressure, which is strongly dependent on the local temperature and the vapour diffusion from the interface to the gaz bulk. Various heat flux distributions are applied along the meniscus to determine conditions under which thermocapillary convection develops. The applied fluxes consider polynomial, Gaussian and combined profiles. It is worth mentioning that the saturated exponential profile (Exp + constant) is the closest one to the realistic profile. The direct numerical simulation allows the the interfacial temperature to be calculed (Fig. 5). It can be noticed that the saturated expression induces a temperature profile close to the one obtained experimentaly by Höhmann \& Stefan [11], exhibiting a minimum value near the contact line. The increases of evaporation near the tube $(r=1)$ induce the appearance of colder region with inversion in temperature gradient sign.

An example of obtained temperature and flow field is shown in Figure 6 for $M a=10$. The isotherms (Fig. 6a) show the non-uniform temperature obtained at the L/V interface with the localised cold region. The heating is assumed via the tube, the evaporation heat extraction is maintained by high temperature gradient near the interface-tube contact and also by the transfer from the bulk via the centre of the domain (axial direction). The temperature gradient induce two counter-rotaing cells, a major one covering the radial extension and small weak remaining one resulting from the strong maintain the tube at uniform and constant temperature. Two regions are distinguished, the first one from the centre to the coldest temperature location where the temperature gradient is supposed to generate a cell rotating from the centre to the contact line. The convective cell developing in second region (near the corner) rotate in the opposite direction. In the experiment work (Sefiane [6]) the second weak cell was not observed, only the main cell rotating from the centre to the contact line has been clearly identified. This can be explained by the fact that

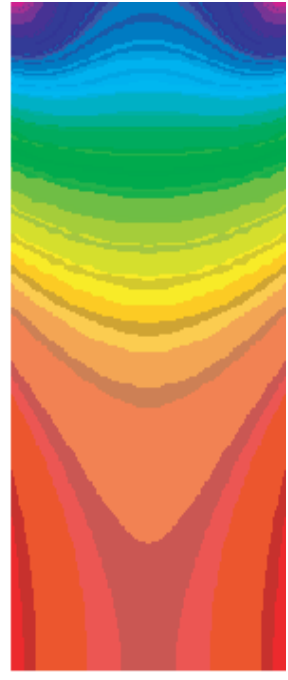

(a)

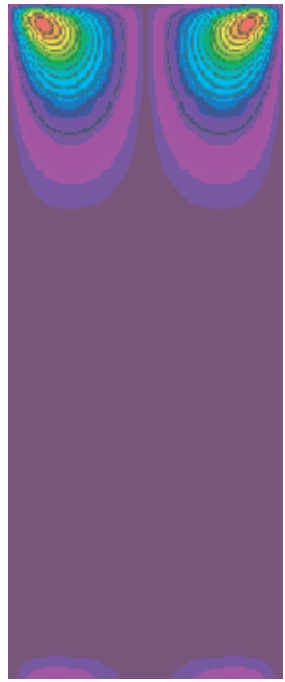

(b)
Fig. 6. Isotherms and streamlines for $M a=10$ (flux profile of the form: $\operatorname{Exp}+$ constant).

friction forces hinder the second convective cell near the wall. This implies that a minimum irregularity in evaporative heat flux will initiate Marangoni convection (Buffone \& Sefiane [12]). The question of a uniform interfacial temperature during evaporation is a question still subject to debate. The evaporation of pure liquids into their vapour, do not exhibit any thernocapillary motion if the system is not contaminated with impurities. The contribution of impurities to the initiation of Marangoni convection in pure systems, indeed could play a major role in some instances as has been discussed by some authors (see Beysens et al. [13]). However the case investigated in this work deals with evaporation of a pure component into air, which introduces a new element that is the role of the local concentration in the gas phase. The configuration investigated suggests a gradient in the local partial pressure because of differential evaporation and diffusion into air. Honda et al. [14] have demonstrated recently that a gradient in the partial pressure indeed exists along the liquid-vapour interface for such systems. Even assuming a saturation temperature (which is dependent on pressure) along the interface, because of the pressure gradient this leads to a temperature gradient along the interface. Using a high sensitivity Infra-Red camera we have been able to capture temperature gradients on the interface of an evaporating meniscus in a capillary tube, results from this investigation will be published subsequently. The partial pressure which is dictated by the diffusive rate into the gas is also related to the evaporative flux, since all evaporated matter has to diffuse into the gas phase. Consequently the temperature gradient establishing along the interface in this specific situation is dictated by the profile of evaporation rate. Another argument in favour of a thermal gradient induced by evaporation, is that it has been shown experimentally that the convection could be reversed by imposing a temperature gradient along the capillary wall in the opposite direction (Buffone et al. [15]). 

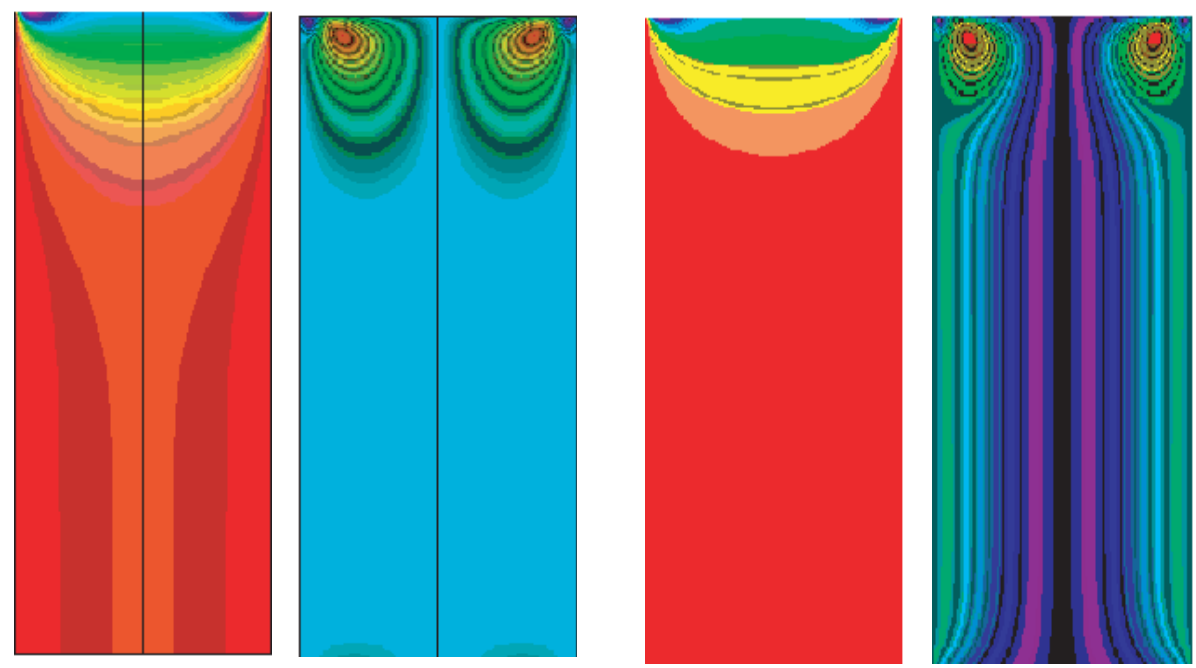

$\mathrm{Pe}=0$

$\mathrm{Pe}=0.1$

Fig. 7. Isotherms and streamlines for $M a=10$ (Gaussian flux profil) for $P e=0$ (a) and $P e=0.1$ (b).

This suggests that in such a configuration the actual driving force for the observed convection is indeed a temperature gradient establishing along the interface induced by differential evaporation.

The presented numerical results are obtained for uniform fixed temperature on the tube, which is a valid assumption for tube of high thermal conductivity (low Biot number). In order to check the weight of such assumption on the obtained result, the following section, boundary conditions are modified to apply the heat flux on the wall of the capillary. In terms of fluid flow only the main convective cell is observed (Figs. 6, 7). The heating conditions don't permit an inversion of the radial temperature gradient responsible of the observed secondary flow in the previous case of imposed temperature. The isotherms show the irregular temperature value obtained on the tube $(r=1)$. Physically increasing $M a$, allows a better mixing in the liquid and tend to decrease the obtained maximum temperature.

As the evaporation induce a global interface motion, such displacement can be handled numerically by referential change described by using the Peclet number $(P e)$. The effect of $P e$ is illustrated in Figure 7. The flow field shows clearly that the main roll is smaller due to the injected fluid. However the temperature field illustrates the reduction depth of the affected cooled area due to evaporation resulting on strong thermal gradient in the reduced space. The aim of the present work was to underline the important dependence between the operating parameters, especially the global motion of the $\mathrm{L} / \mathrm{V}$ interface $(P e)$ and the local evaporation rate, in improving the efficiency of considered heat pipes.

\section{Conclusion}

The paper provides a survey on heat pipes structure and operating principles allowing alternatives to cool electronic components. Heat pipes take the advantage of phase change and capillary force transportation to achieve high heat transfer rates. For such devices experimental access to the local evaporation rate is difficult, and the numerical simulation can be an effective tool to describe the controlling parameters. The present work analyses the necessary conditions allowing the apparition of convective motion and its interaction with energy exchange at the liquid/vapour interface. A computational model successfully used for CFD problems involving liquid bridge is extended to capillary tube configurations.

The capillary tubes considered for the present study are characterized by a small aspect ratio allowing the study to neglect the buoyancy convection and to focus on thermocapillary one. Various heat flux distributions are applied along the meniscus and conditions allowing the development of thermocapillary convection are characterised, the study permits to select a profile close to the realistic experimental observations. It is shown that the inversion of the distribution is responsible of the development and the right orientation of the Marangoni cells.

The simulation allows the access to the interfacial temperature and to characterise its interaction with $M a$ convection. Various Marangoni number values are considered to show flow patterns and temperature distribution. Diffusive and convective regimes are investigated with describing local convection near the liquid vapour surface.

\section{References}

[1] K. Azar, Managing power requirements in the electronics industry, Electronic Cooling Magazine 6(4) (2000)

[2] S. Berestecky, Thermal design of fault tolerant and high availability computer systems, Electronics Cooling Magazine 5(3) (1999)

[3] R.C. Chu, A review of IBM sponsored research and development projects for computer cooling, Proceedings of 15th IEEE Semi-Therm Symposium, 1999, pp. 151-165 
[4] S.D. Garner, Heat pipes for electronics cooling applications, Electronic Cooling Magazine (1996)

[5] J.E. Graebner, Heat pipe fundamentals, Electronics Cooling Magazine 5(2) (1999)

[6] K. Sefiane, A. Steinchen, On the thermocapillary effects in the evaporation of a meniscus from a capillary tube, Proceeding of the 12th Int. Heat and Mass Transfer Conf., 2002, pp. 437-441

[7] T. Molenkamp, Marangoni Convection, Mass Transfer and Microgravity, Ph.D. thesis, University of Groningen (1998)

[8] O.E. Ruiz, W.Z. Black, Evaporation of water droplets placed on a heated horizontal surface, J. Heat Transfer ASME 124(5) (2002) 854-863

[9] S. Pantakar, Numerical heat transfer and fluid flow, Hemisphere, New York, 1980

[10] R. Bennacer, A.A. Mohamad, E. Leonardi, Computational analysis of Marangoni effects during floating zone growth under microgravity conditions in heat transfer, Numerical Heat Transfer, Part A 41 (2002) $657-671$
[11] C. Höhmann, P. Stephan, Microscale temperature measurement at an evaporating liquid meniscus, Experimental Thermal and Fluid Science 26(2-4) (2002) 157-162

[12] C. Buffone, K. Sefiane, Marangoni Convection in Capillary Tubes Filled with Volatile Liquids, First International Conference on Microchannels and Minichannels, ASME, Rochester, NY, USA (2003)

[13] D. Beysens, Y. Garrabos, V.S. Nikolayev, C. Lecoutre, J.-P. Delville, J. Hegseth, Liquid-vapor phase separation in a thermocapillary force field, Europhysics Letters 59(2) (2002) 245-251

[14] H. Honda, Y.S. Wang, Stratified flow model of evaporation heat transfer in horizontal microfin tubes, 8th UK Heat Transfer Conference, Oxford, Sep. (2003), submitted to the Inter. J. Heat and Mass Transfer

[15] C. Buffone, K. Sefiane, J.R.E. Christy, Experimental investigation of the hydrodynamics and stability of an evaporating wetting film placed in a temperature gradient, Proc. of the UK Heat Transfer Conference, Oxford, Sep. (2003), to appear in Applied Thermal Engineering

To access this journal online: www.edpsciences.org/meca 